\title{
KLOTHO DEFICIENCY PROMOTES THE PROLIFERATION AND MIGRATION OF HUMAN AORTIC VASCULAR SMOOTH MUSCLE CELLS BY ACTIVATING THE WNT/B-CATENIN SIGNALING PATHWAY
}

\author{
Jian-Hang Wei', Quan Liang1, Bo Gao², Yun Pan², Li-Hua Li'* \\ 1 Department of Gerontology, The First Affiliated Hospital of Dali University, Dali 671000, Yunnan Province, China \\ 2 Department of Pathology, The First Affiliated Hospital of Dali University, Dali 671000, Yunnan Province, China \\ E-mail: lilihuayncn@163.com
}

\begin{abstract}
Objective: Arterial stiffness is a component of arterial aging and might be related to decreased expression of Klotho. We performed Klotho RNA interference in human aortic vascular smooth muscle cells (HA-VSMC) to assess the effects of Klotho silencing on cell proliferation, cell migration, and the Wnt signaling pathway. Methods: Klotho expression in HA-VSMC was reduced by RNA interference. Cell proliferation and migration were determined by Cell Counting Kit-8 (CCK-8) assay and transwell assay, respectively.

Results: Decreased expression of Klotho promotes the proliferation and migration of HA-VSMC. Furthermore, we found that decreased expression of Klotho led to the activation of the Wnt/ $\beta$-catenin signaling pathway, with increased expression of multiple Wnt protein isoforms and $\beta$-catenin accompanied by increased expression of TCF 4 and Cyclin DI, which are downstream target proteins of the $\mathrm{Wnt} / \beta$-catenin signaling pathway. Additionally, the Wnt signaling pathway inhibitor XAV939 significantly inhibited the proliferation of HA-VSMC treated with shKlotho in a dose-dependent manner.

Conclusion: Klotho deficiency promotes HA-VSMC proliferation and migration, and the Wnt/ $\beta$-catenin signaling pathway may play an important role in the process of arterial stiffness related to vascular aging.
\end{abstract}

Keywords: Arterial stiffness, human vascular smooth muscle cell, Klotho, Wnt signaling, XAV939 


\section{Significance Statement}

Arterial stiffness is a component of arterial aging and might be related to decreased expression of Klotho. We performed Klotho RNA interference in human aortic vascular smooth muscle cells (HA-VSMC) to assess the effects of Klotho silencing on cell proliferation, cell migration, and the Wnt signaling pathway. Klotho expression in HA-VSMC was successfully reduced by RNA interference, and we found that decreased expression of Klotho promotes the proliferation and migration of HA-VSMC. Furthermore, we found that decreased expression of Klotho led to the activation of the Wnt/ $\beta$-catenin signaling pathway, and the Wnt signaling pathway inhibitor XAV939 significantly inhibited the proliferation of HA-VSMC treated with shKlotho in a dose-dependent manner.

\section{Introduction}

Cardiovascular disease (CVD) is the leading cause of death in China and accounts for $40 \%$ of deaths in the Chinese population [1]. Among the risk factors of CVD, hypertension is the most critical risk factor in China; an estimated $43 \%$ of CVD events can be attributed to hypertension $[2,3]$. Indeed, the prevalence of hypertension in the Chinese population aged $\geq 18$ years during October 2012 to December 2015 was $23.2 \%$, accounting for about 240 million patients [4]. Aging is a significant risk factor for hypertension and CVD. Vascular aging, an essential aspect of human aging, leads to intimal and medial thickening as well as the gradual loss of arterial elasticity resulting in arterial stiffness. Furthermore, arterial stiffness is the primary cause of hypertension and pathophysiological processes related to aging [5]. Arterial stiffness may not only be a significant cause of hypertension but may also play a role in target organ damage $[6,7]$. One study has shown that severe arterial stiffness is the leading cause of poor efficacy of hypertension drugs. Most of the current antihypertensive drugs are neuroendocrine blocking agents, and there are no drugs that can effectively improve arterial elasticity [8].

Klotho is an anti-aging protein mainly expressed in the renal distal convoluted tubules and choroids [9, 10]. Klotho can exist as a membrane-bound protein that can be cleaved and released as a soluble protein in the blood, urine, and cerebrospinal fluid. Circulating Klotho declines with aging [10]. Previous studies have indicated that Klotho deficiency could promote medial calcification, intima hyperplasia, endothelial dysfunction, arterial stiffening, hypertension, and impaired angiogenesis and vasculogenesis, which are characteristics similar to those of aged human arteries [11-17]. However, the underlying mechanisms by which Klotho affects arterial stiffness are mostly unknown, and the mechanisms of Klotho deficiency in rat and human arterial stiffness might not be identical.

Vascular smooth muscle cells (VSMC) play a central role in the pathophysiologic process of vascular remodeling [18]. VSMC can perform both contractile and synthetic functions, which are characterized by differences in morphology, proliferation, and migration. VSMC play a key role in 
many cardiovascular diseases such as atherosclerosis, restenosis, and hypertension [19]. Previous studies mainly studied Klotho deficiency or overexpression on animal VSMC calcification, proliferation, migration, and oxidative stress [20-22]. In kidney tissue of chronic kidney disease models, it has been shown that Klotho expression is inversely related to $\beta$-catenin expression, and that Klotho can inhibit Wnt-induced $\beta$-catenin activation, nuclear translocation, and target gene expression [23]. However, the effect of Klotho deficiency on the proliferation and migration of human VSMC and its underlying mechanisms are unclear.

In this study, we successfully reduced the expression of Klotho in human aortic VSMC (HA-VSMC) through RNA interference technology and investigated the effects of Klotho deficiency on the proliferation and migration of HA-VSMC. Specifically, we investigated whether the Wnt/ $\beta$-catenin signaling pathway plays a key role in the response of HA-VSMC to Klotho deficiency, and used a small molecule inhibitor of the Wnt/ $\beta$-catenin signaling pathway (XAV939) to confirm our results.

\section{Methods}

\section{Materials}

Human aortic smooth muscle cells (BeNa Culture Collection); Primer

(Sangon Biotech); Protease inhibitor cocktail (Promega); Goat Anti-Mouse IgG and

Goat Anti-Rabbit IgG (CWBIO); $\beta$-catenin, Cyclin D1 and Klotho antibodies (Abcam); Wnt3A antibody (OriGene); Protease inhibitor cocktail (Promega); Cell Counting Kit-8 CCK-8 (Meilunbio); Transwell nesting chamber $16.5 \mathrm{~mm}$ transwell with $8.0 \mu \mathrm{m}$ pore polycarbonate membrane, Corning); XAV939 (Selleck).

\section{Cell Culture and Transfection}

HA-VSMC were cultured in high glucose DMEM medium containing $15 \%$ FBS. The incubator temperature was $37^{\circ} \mathrm{C}$ and the $\mathrm{CO}_{2}$ concentration was $5 \%$. Lentiviral shRNA (GenePharma) was used to interfere with Klotho expression. The Klotho-2073 shRNA (shKlotho-2073) target sequence was 5'-GAGCCGTATACAAGGAATATG-3'; the Klotho-1021 target shRNA (shKlotho-1021) sequence was 5'-CCGAGAGCATGAAGAATAACC-3'; the Klotho-1207 shRNA (shKlotho-1207) target sequence

was

5'-GGATTGACCTTGAATTTAACC-3'; and the negative control shRNA-NC (shNC) target sequence was 5'-TTCTCCGAACGTGTCACGT-3'. The cells were seeded in a 6 -well plate at $3 \times 105 /$ well, and the cells were treated with $1 \mu \mathrm{g} / \mathrm{mL}$ of polybrene and lentiviral suspension ( $\mathrm{MOI}=$ 20) after 1 day of cell confluence. After 72 hours of transfection, the virus solution was discarded, and cells were used for experiments.

\section{Proliferation Assay Using Cell Counting Kit-8}

We inoculated the cells at $5 \times 10^{3}$ cells/well in 96-well plates, each of which was repeated 5 times while setting blank control wells. Cell Counting Kit-8 (CCK-8) was observed at 0 h, 24 h, 48 h, 72 h, 96 h, and $120 \mathrm{~h}$. Before the test, $10 \mu \mathrm{l}$ of CCK-8 solution was added to each well. The cells were incubated for $2 \mathrm{~h}$, and the 
absorbance (wavelength: $450 \mathrm{~nm}$ ) was established on the enzyme marker.

According to the instructions, $10 \mathrm{mg}$ of XAV-939 was dissolved in $1 \mathrm{~mL}$ of DMSO, yielding a concentration of $10 \mathrm{mmol} / \mathrm{L}$. The original solution was diluted into culture medium at concentrations of $0 \mu \mathrm{mol} / \mathrm{L}, 5$ $\mu \mathrm{mol} / \mathrm{L}, 10 \mu \mathrm{mol} / \mathrm{L}, 20 \mathrm{\mu mol} / \mathrm{L}, 50 \mathrm{\mu mol} / \mathrm{L}$, and $100 \mu \mathrm{mol} / \mathrm{L}$. We inoculated the cells at $5 \times 10^{3}$ cells/well in 96-well plates, each of which was repeated 5 times while setting blank control wells. Cell Counting Kit-8 (CCK-8) was observed at $24 \mathrm{~h}$ and $48 \mathrm{~h}$. Prior to testing, $10 \mu \mathrm{L}$ of CCK-8 solution was added to each well. The cells were incubated for 2 hours, and absorbance was established on the enzyme label (wavelength: $450 \mathrm{~nm}$ ).

\section{Real-time PCR}

CDNA was synthesized by reverse

\begin{tabular}{ccc}
\hline Gene & & Primer sequence (5'-3') \\
& Forward & TGCACCACCAACTGCTTAGC \\
GAPDH & Reverse & GGCATGGACTGTGGTCATGAG \\
& Forward & CTGGATGGTATCAATCTTTGCG
\end{tabular}

Klotho

Reverse ATCTGCAGCATAACGATAGAGG

Forward CCGATGGTGGGGTATTGTGAA

Wnt1

Reverse TCCCCGGATTTTGGCGTAT

Forward AGTCGGGAATCTGCCTTTGTT

Wnt2

Reverse TGGCGCTTCCCATCTTCTTT

Forward TTGTGAGCCCAACCCAGAGA

Wnt3 transcription on ice according to the kit instructions (FastKing RT Kit With gDNase, TIANGEN). Real-time PCR amplification was performed using this cDNA as a template. The expression levels of Klotho, Wnt1, Wnt2, Wnt3, Wnt3A, Wnt4, Wnt5A, Wnt5B, Wnt7A, Wnt9A, Wnt9B, Wnt10B, TCF4, Cyclin D1, and $\beta$-catenin mRNA were detected, and GAPDH was used as an internal reference. The relative expression levels of each group were collected, analyzed, and expressed as $2^{-} \Delta \Delta \mathrm{C}+$ values.

We treated cells with both XAV939 and Klotho RNA interference, extracted RNA, and determined the concentration to synthesize cDNA. Using this cDNA as a template, real-time PCR amplification was performed, and the relative expression of Wnt9A was detected, calculated, and statistically analyzed.

\section{Product Size (bp)}

$87 \mathrm{bp}$

$90 \mathrm{bp}$

$137 \mathrm{bp}$

$124 \mathrm{bp}$

$138 \mathrm{bp}$

Reverse TTTTCCTTCCGCTTCTCCGT 


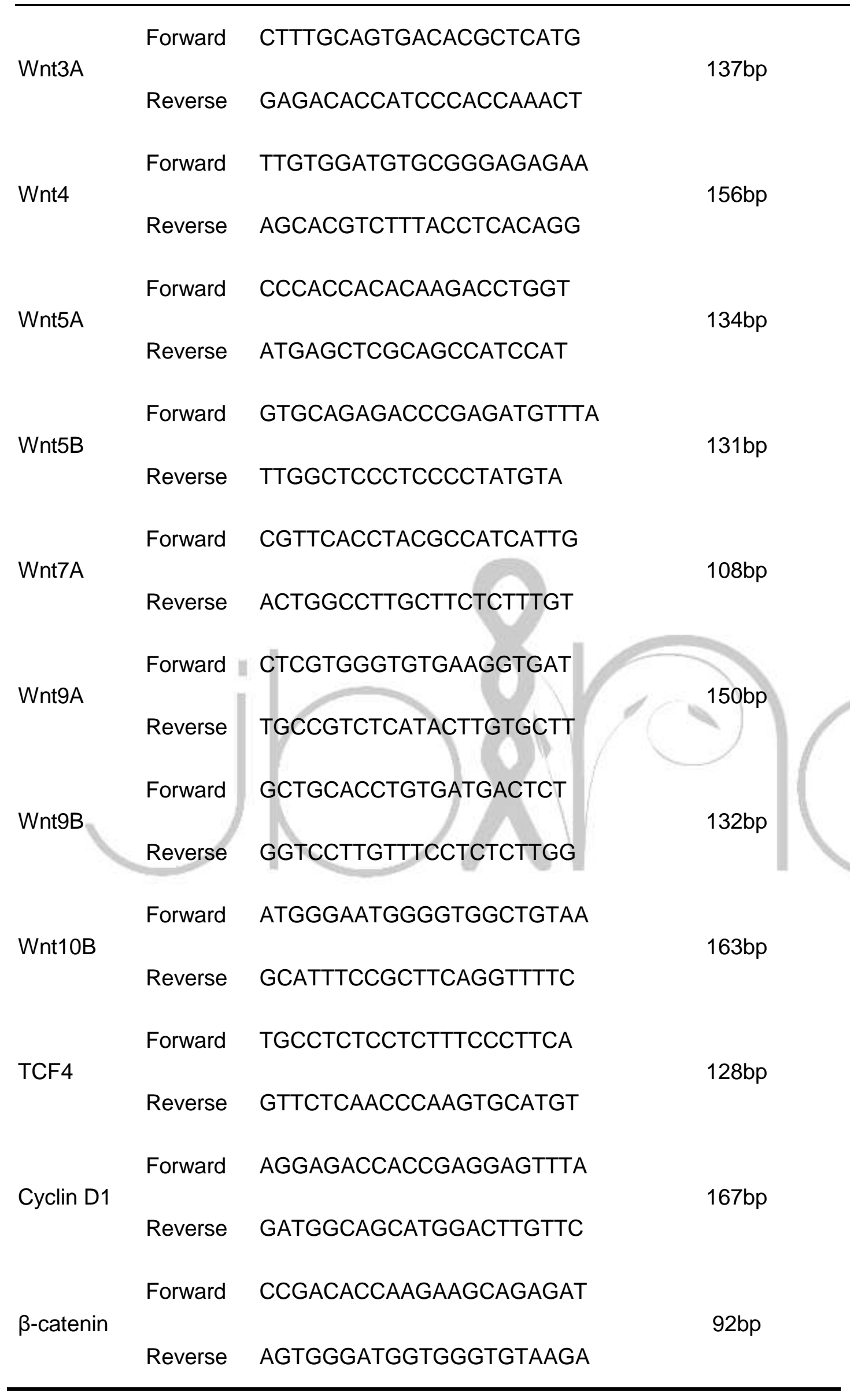




\section{Western Blots}

Western blot analysis was performed to study protein expression in HA-VSMC. The total HA-VSMC protein content was isolated using a western blotting lysis kit (Protease inhibitor cocktail, Promega) and separated by $10 \%$ SDS-polyacrylamide gel electrophoresis (PAGE). Proteins were then transferred onto a polyvinyl difluoride (PVDF) membrane and blocked with $5 \%$ dried milk for $2 \mathrm{~h}$. The primary antibody (1:1000 Tubulin, 1:1000 anti-klotho, 1:1000 anti-Wnt3A, 1:5000 anti- $\beta$-catenin and 1:1000 anti-Cyclin D1) was diluted in TBS-T containing 5\% skim milk, and was then added to the membrane and incubated at $4^{\circ} \mathrm{C}$ overnight. After three washes with TBS-T, the secondary antibody conjugated to horseradish peroxidase 11:2000 Goat Anti-Mouse IgG, 1:2000 Goat Anti-Rabbit IgG) was added to the membrane and incubated at room temperature for $2 \mathrm{~h}$. Immunoblots were developed on films using the Bio-Rad gel imaging system. The optical density analysis of the bands was determined using Image $J$ and assayed with Tubulin correction for relative amount analysis.

\section{Transwell migration assay}

Cells were digested, centrifuged, and washed with PBS once. Then, cell pellets were suspended in DMEM medium without serum, and cells were counted and the cell concentration was adjusted. We added $200 \mu \mathrm{L}$ cell suspension $\left(2 \times 10^{5}\right.$ cells) to the upper chamber of each Transwell plate, and added $500 \mu \mathrm{l}$ medium containing 15\%
FBS into the lower chamber of each Transwell plate. Plates were incubated in a $37^{\circ} \mathrm{C}$ and $5 \% \mathrm{CO}_{2}$ incubator. After $48 \mathrm{~h}, 72$ $\mathrm{h}$, and $96 \mathrm{~h}$ of incubation, plates were fixed, stained, and dried. Ten different visual fields were randomly selected to calculate the number of transmembrane cells.

\section{Statistical Analysis}

Data analysis was performed using SPSS 23.0 software. Measurement data are expressed as mean \pm standard deviation, and two sets of data were compared using two independent sample t-tests; multiple samples were compared using analysis of variance. When $P<0.05$, the difference was considered statistically significant.

\section{Results}

Our fluorescence microscopy and quantitative fluorescence PCR experiments showed that the expression of Klotho was significantly inhibited by shKlotho-2073. The knockdown efficiency was $86.4 \%$ (Fig. 1A). Thus, shKlotho-2073 (shKlotho) was chosen as the interference vector. The results were further verified by Western blot (Fig. 1B, 1C).

The CCK-8 tests showed that the proliferation of shKlotho-transfected HA-VSMC was significantly higher than that of the control group, and the difference was more obvious with increasing experimental time (Fig. 2A). The comparison of each time point from $24 \mathrm{~h}$ was statistically significant ( $P<0.001)$. Throughout the four-day-long Transwell migration experiment, the migration ability of shKlotho-transfected HA-VSMC was significantly increased. The results show that the shKlotho group has a statistically 
higher number of transmembrane cells after $72 \mathrm{~h}$ compared with the shNC group $(\mathrm{P}<0.01$ ) (Fig. 2B).

After the transfection of HA-VSMC with shKlotho, we further investigated whether the Wnt/ $\beta$-catenin signaling pathway was affected. Using RT-PCR and Western blot analyses, we found that the relative mRNA expression of Wnt1, Wnt2, Wnt3, Wnt3A, Wnt4, Wnt5A, Wnt5B, Wnt7A, Wnt10B, and especially Wnt9A and Wnt9B was increased significantly (Fig. 3A, 3B). The results of Western blot analyses of Wnt3A (Fig. 3D, 3G) indicated that its expression in the shKlotho group was significantly higher than that in the shNC group ( $n=3,2.441$ vs. 1.870, $\mathrm{P}=0.0003)$. The relative mRNA ( $n=3$, Fig. $3 C$ ) and protein expression ( $n=3$, Fig. $3 F, 31)$ of $\beta$-catenin was significantly higher in the shKlotho group than in the shNC group. These results indicate that Klotho deficiency can activate the Wnt/B-catenin signaling pathway. In addition, after transfection with shKlotho, we detected a significant increase in TCF4 expression at the mRNA level (Fig. 3C). RT-PCR and Western blot also confirmed that the expression of Cyclin DI was increased significantly (Fig. 3C, 3E, 3H).

The results of the CCK-8 experiments showed that the Wnt signaling pathway inhibitor XAV939 significantly inhibited the proliferation of normal HA-VSMC in a dose-dependent manner (Fig. 4A). After shklotho treatment, XAV939 also significantly inhibited the proliferation of HA-VSMC in a dose-dependent manner (Fig. 4B, 4C). After treating HA-VSMC with
shKlotho, cells were cultured for 48 hours in normal medium, media containing 50 umol/L XAV939, and media containing 100 $\mu \mathrm{mol} / \mathrm{L}$ XAV939. We found that XAV939 significantly inhibited the expression of Wnt9A at the mRNA level in HA-VSMC treated with shKlotho (Fig. 4D).

\section{Discussion}

Arterial stiffness and hypertension are aging-related cardiovascular disorders. It is important to investigate how vascular aging, a process characterized by gradual Klotho deficiency, participates in arterial stiffness and hypertension. It is well known that excessive VSMC proliferation and migration are primary mechanisms of vascular remodeling. In the present study, we systematically assessed the effect of Klotho deficiency on the $\mathrm{Wnt} / \beta$-catenin signaling pathway and VSMC proliferation and migration. We found that shKlotho intervention significantly reduced the expression of Klotho, and subsequently increased proliferation and migration of VSMCs via activating the $\mathrm{Wnt} / \mathrm{\beta}$-catenin pathway.

Previous studies have shown that the anti-aging protein Klotho plays an important role in vascular endothelial dysfunction, VSMC proliferation and migration, monocyte chemotaxis, and vascular tissue inflammation [24,25]. Klotho is involved in vascular protection via inhibition of oxidative stress, regulation of inflammation, and reduction of vascular calcification [12,26-28]. The results of the present study are consistent with those of previous studies. In rat VSMC, recombinant 
exogenous Klotho regulates BAG3 the expression of proliferating proteins, and expression through the NF-KB p65 pathway changed the cytoskeleton by inhibiting the and inhibits the proliferation and migration NF-KB, P65, Akt, and ERK signaling of VSMC induced by Angiotensin II (Ang II) pathways. Therefore, Klotho can effectively [29]. Yu et al. [21] found that Ang II can promote the proliferation and migration of VSMC, reduce SM22a, and promote the expression of PCNA, suggesting that Ang II can regulate the phenotype of VSMCs. Co-treatment with Klotho increased the expression of contractile proteins, reduced inhibit Ang II-induced VSMC proliferation, migration, and phenotypic regulation [21]. In this study, we confirmed that low expression of Klotho can promote the proliferation and migration of HA-VSMC, which may be involved in the occurrence and development of arteriosclerosis.

Fig 1. Klotho silencing in HA-VSMC. (A) Detection of Klotho transfection efficiency at the mRNA level. (B, C) Changes in protein expression after shKlotho interference in HA-VSMC. ${ }^{*} \mathrm{P}<0.05,{ }^{* *} \mathrm{P}<0.01,{ }^{* *} \mathrm{P}<0.001$.
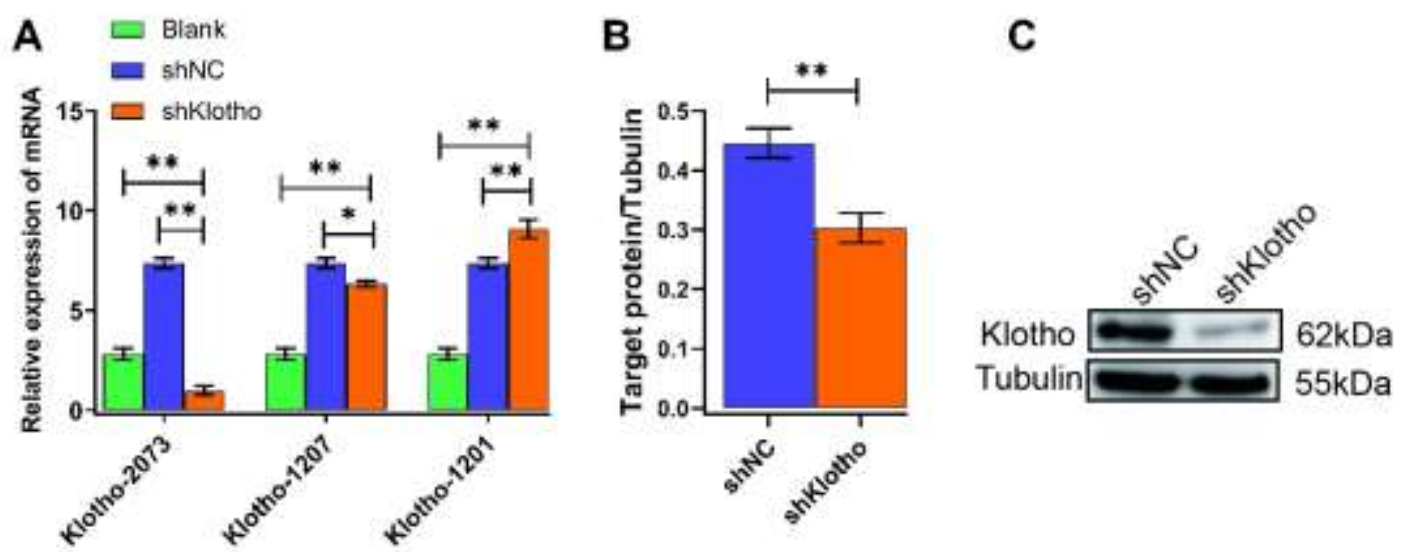
Fig 2. shKlotho interference significantly promotes the proliferation and migration of HA-VSMC. (A) CCK-8 detected the proliferation of shKlotho-treated HA-VSMC. (B) Changes in migration at different time points after shKlotho treatment in HA-VSMC. ${ }^{*} \mathrm{P}<0.05,{ }^{* *} \mathrm{P}<0.01,{ }^{* *} \mathrm{P}<0.001$.

A

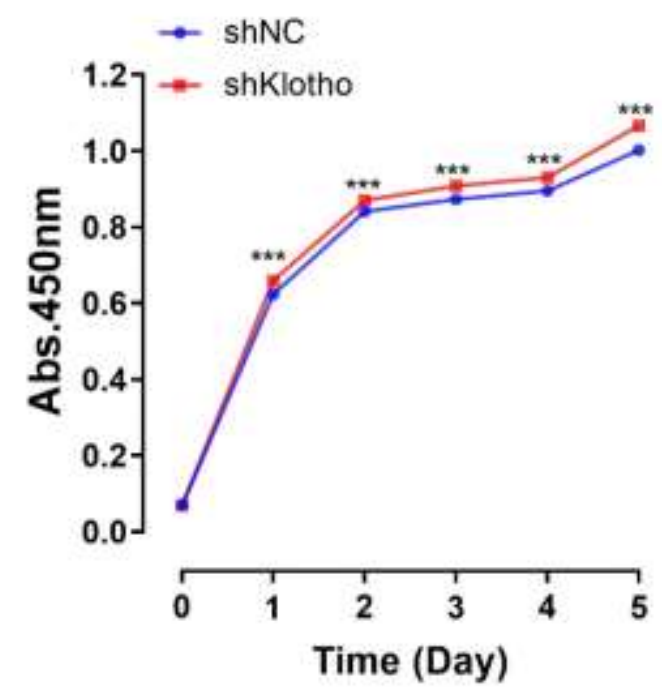

B

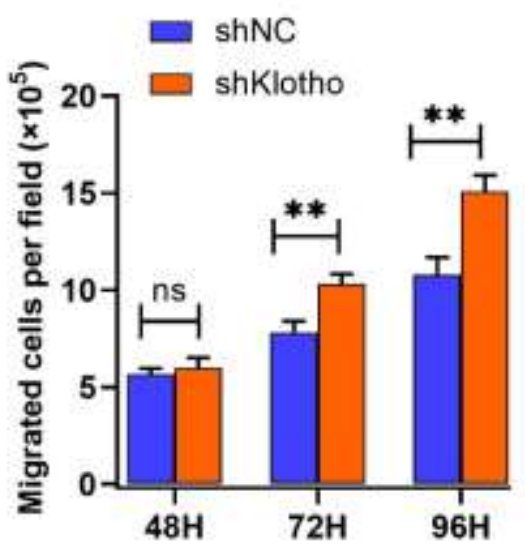


Fig 3. shKlotho interference on HA-VSMC activates the Wnt signaling pathway. (A, B) Changes in Wnt family protein expression after shKlotho transfection. (C) $\beta$-catenin, TCF-4 and cyclin D1 expression after shKlotho transfection. (D, G) Wnt3A protein expression after shKlotho transfection. (E, H) Cyclin D1 protein expression after shKlotho transfection. $(\mathbf{F}, \mathbf{I}) \beta$-catenin protein expression after shKlotho transfection. ${ }^{*} \mathrm{P}<0.05,{ }^{* *} \mathrm{P}<0.01,{ }^{* *} \mathrm{P}<0.001$.
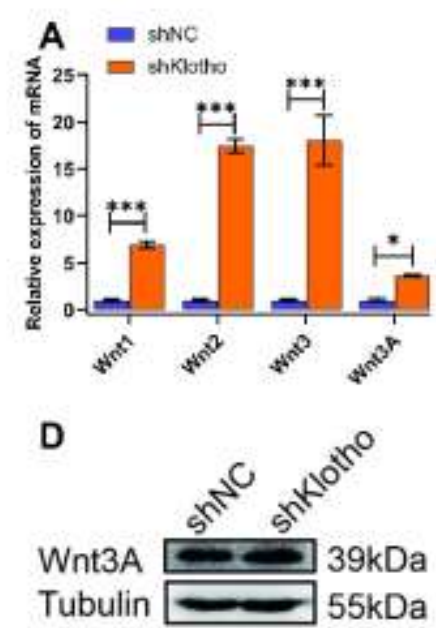

G

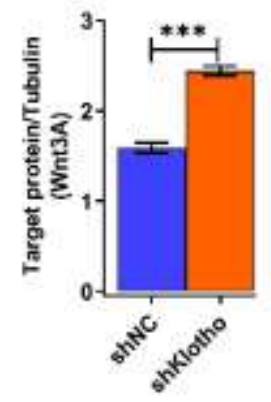

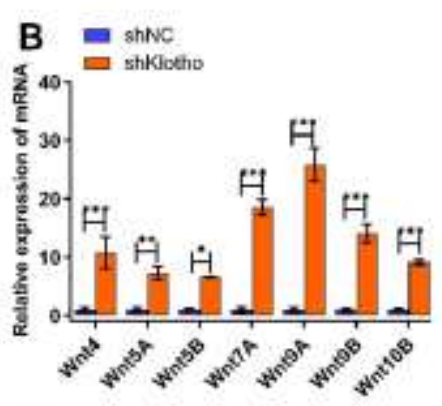

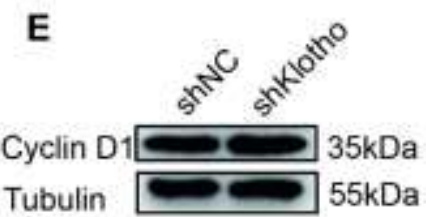

H

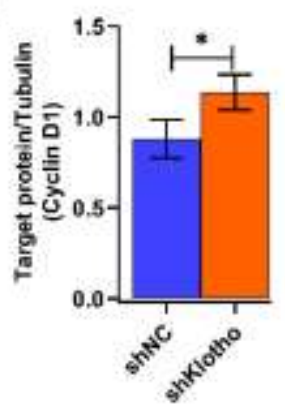

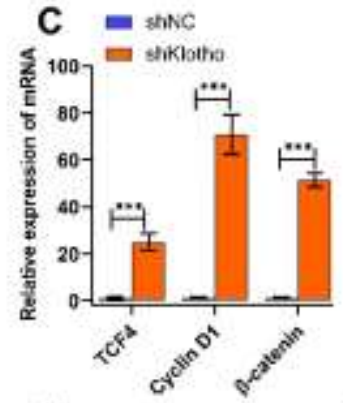
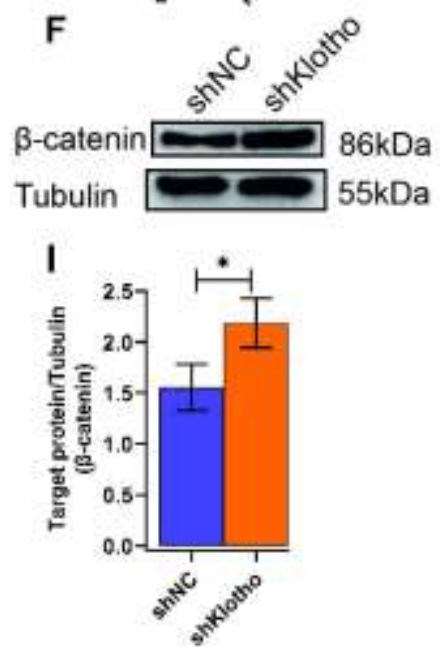
Fig 4. Treatment of HA-VSMC with XAV939 inhibits proliferation and Wnt9A expression. (A) CCK-8 experiments showing that XAV939 significantly inhibits the proliferation of normal HA-VSMC in a dose-dependent manner. (B, C) Proliferation of HA-VSMC after shKlotho and XAV939 treatment. (D) RT-PCR measurement of Wnt9a mRNA expression after shKlotho and XAV939 treatment. * $P<0.05$, ${ }^{* *} \mathrm{P}<0.01,{ }^{* *} \mathrm{P}<0.001$.
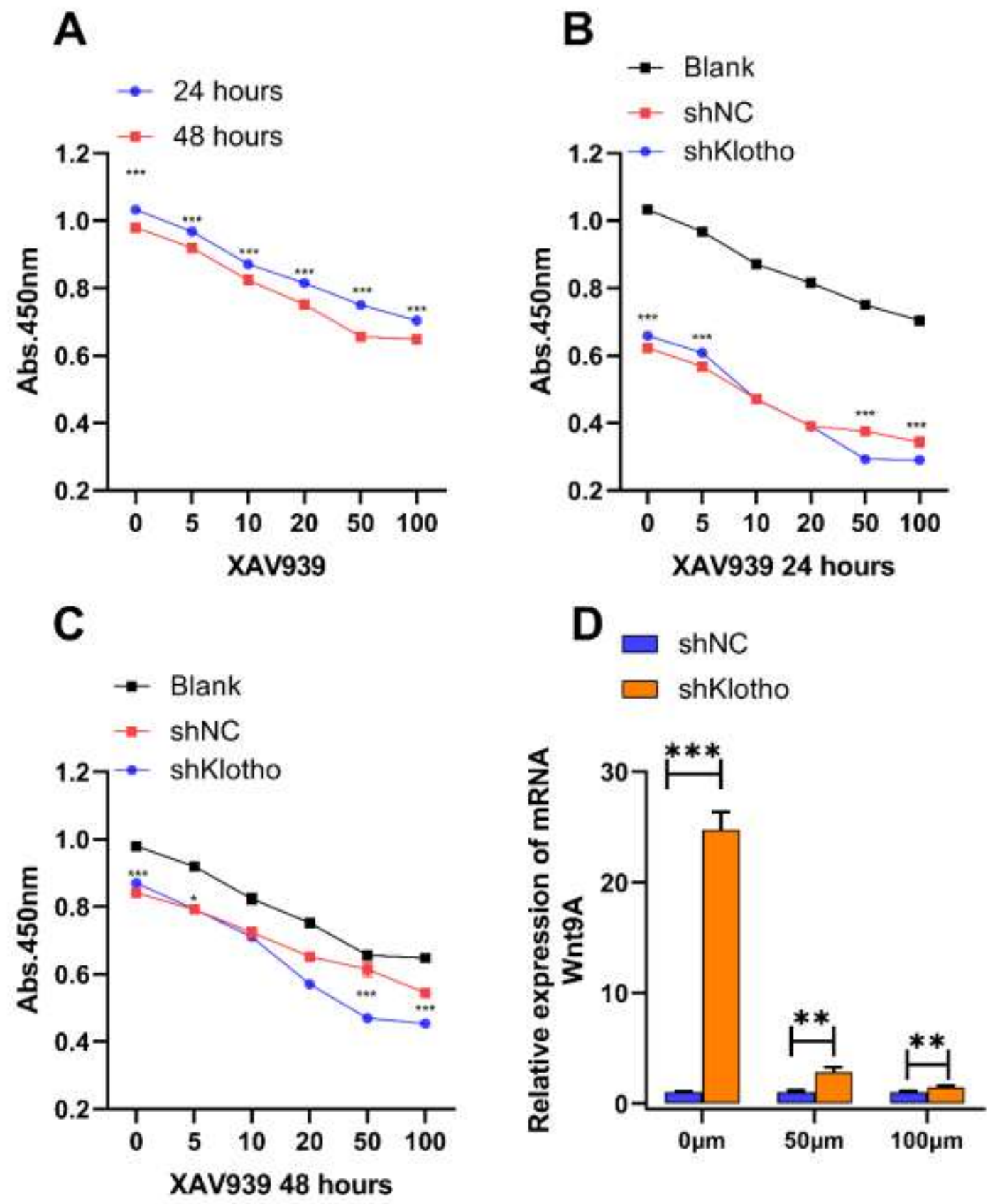

Klotho is an endogenous inhibitor of the pressure, kidney injury, hypertensive heart Wnt/ $\beta$-catenin signaling pathway, which is disease, and type 2 cardiorenal syndrome widely involved in the regulation of blood [30-33]. Depending on the Wnt protein and 
the downstream receptor protein, the Wnt signaling pathway is divided into a classical signaling pathway (Wnt/ß-catenin signaling pathway) and a non-canonical signal (the planar cell polarity pathway and Wnt/Ca ${ }^{2+}$ signaling pathway) [10]. The classical Wnt signaling pathway is dependent on intracellular $\beta$-catenin. When the Wnt protein interacts with the transmembrane Frizzled receptor Frizzled (Fzd) and the co-receptor, low-density lipoprotein (LDL) receptor-associated protein (LRP5/6), a series of downstream signaling events are induced [34]. Eventually, $\beta$-catenin is translocated into the nucleus and binds to the nuclear transcription factor, $T$ cell factor (TCF)/lymphoid enhancer-binding factor (LEF), to stimulate transcription of the Wnt target gene. Zhou et al. found that Klotho expression was negatively correlated with $\beta$-catenin in kidney tissues of animal models of chronic kidney disease [35]. In vitro experiments showed that either membrane Klotho or secretory Klotho could bind to Wnt proteins and inhibit Wnt-induced $\beta$-catenin activation, nuclear translocation, and target gene expression. When the Wnt signal is absent, $\beta$-catenin in the cytoplasm is degraded by the proteasome. The activation of the Wnt signaling pathway will result in the accumulation of $\beta$-catenin in the cytoplasm and then translocation into the nucleus. $\beta$-catenin interacts with transcription factors such as those in the T-cytokines (TCF) family to activate target genes such as TCF4 and Cyclin DI and lead to abnormal cell proliferation. It has been found that the extracellular segment of Klotho can bind to some Wnt ligands and block the binding of Wnt to its corresponding cell surface receptors, thus inhibiting the Wnt signaling pathway [36]. In the present study, we investigated the regulatory effect of Klotho on the Wnt/ $\beta$-catenin signaling pathway in HA-VSMC. Our results showed that silencing of Klotho enhanced the activation of the Wnt/ $\beta$-catenin signaling pathway, suggesting that Klotho could activate the Wnt/ $\beta$-catenin signaling pathway in arterial stiffness. However, we do not know whether the HA-VSMC response to Klotho silencing depends solely on the Wnt/ $\beta$-catenin signaling pathway or if other signaling pathways are involved. Further study is needed.

A variety of diseases are currently associated with abnormal activation of the Wnt signaling pathway, and targeted therapy for Wnt signaling has become a research hotspot [37]. Chen et al. treated rat VSMCs with XAV939, and found that XAV939 inhibits the formation of intima, which is manifested by the loss of intimal area and the I/M ratio, as well as by weakened proliferation and migration capabilities, increased ROS production, and promotion of cell cycle arrest in VSMCs. They therefore confirmed that XAV939 attenuates the formation of the intima by inhibiting the Wnt signaling pathway and inhibiting VSMC proliferation, migration, and apoptosis [38]. Studies have shown that Klotho significantly inhibits Ang Il-induced cardiac hypertrophy and $\beta$-catenin pathway activation. The use of 
XAV939 can inhibit Ang Il-induced $\beta$-catenin activity, expression of the $\beta$-catenin target genes c-myc and Cyclin D1, and Ang Il-induced cardiac 2 . hypertrophy [39].

To our knowledge, this is the first study to systematically demonstrate Klotho deficiency promotes proliferation and migration in HA-VSMC via Wnt/B-catenin signaling pathway activation. Our finding is significant because it points to a new direction for understanding the 3. pathogenesis of aging-related arterial stiffness. Our study may offer novel insights for using the antiaging protein Klotho or small molecule Wnt/ $\beta$-catenin inhibitors as new therapeutic agents to treat arterial stiffness and hypertension.

\section{Acknowledgments}

The Chinese Ministry of Science and Technology (81460084, 81660072 , 81860084), Young and Middle-aged Academic Leader Training Foundation of Yunnan Province (2015HB056), Medical 5. Academic Leader Foundation of Yunnan Provincial Bureau of Health (D-201672), Innovation Team of Hypertension Prevention and Treatment of Dali University (ZKPY2019304), Ten-thousand Talents Program of Yunnan Province, and Yunnan Key Lab of Pathology supported this research. We would like to thank LetPub (www.letpub.com) for providing linguistic assistance during the preparation of this manuscript

\section{References}

1. Chen W, Gao R, Liu L, Zhu M, Wang W,
Wang Y. Report on cardiovascular diseases in China (2017, summary). Chin Circ J 2018; 33: 1-8.

Collaborators GDH. Global, regional, and national disability-adjusted life years (DALYS) for 306 diseases and injuries and healthy life expectancy (HALE) for 188 countries, 1990-2013: quantifying the epidemiological transition. Lancet 2015; 386: 2145-2191.

\section{Zhang G, Yu C, Zhou M, Wang L, Zhang Y,} Luo L. Burden of Ischaemic heart disease and attributable risk factors in China from 1990 to 2015: findings from the global burden of disease 2015 study. BMC Cardiovasc Disord 2018; 18: 18.

4. Wang Z, Chen Z, Zhang L, Wang X, Hao G, Zhang $\mathbf{Z}$, et al. Status of Hypertension in China: Results From the China Hypertension Survey, 2012-2015. Circulation 2018; 137: 2344-2356.

Mitchell GF, Parise H, Benjamin EJ, Larson MG, Keyes MJ, Vita JA, et al. Changes in arterial stiffness and wave reflection with advancing age in healthy men and women: the Framingham Heart Study. Hypertension 2004; 43: 1239-1245.

Kaess BM, Rong J, Larson MG, Hamburg NM, Vita JA, Levy D, Benjamin EJ, Vasan RS, Mitchell GF. Aortic stiffness, blood pressure progression, and incident hypertension. JAMA 2012; 308: 875-881.

Mitchell GF. Arterial stiffness and hypertension. Hypertension 2014; 64: 13-18. 
protein-1/CC chemokine receptor

Protogerou A, Blacher J, Stergiou GS, Achimastos A, Safar ME. Blood pressure response under chronic antihypertensive drug therapy: the role of aortic stiffness in the REASON (Preterax in Regression of Arterial Stiffness in a Controlled Double-Blind) study. J Am Coll Cardiol 2009; 53: $445-451$.

\section{Kuro-o M, Matsumura $Y$, Aizawa $H$,} Kawaguchi $H$. Mutation of the mouse klotho gene leads to a syndrome resembling ageing. Nature 1997; 390: 45-51.

Kuro-o M. Klotho and aging. Biochim Biophys Acta Gen Subj 2009; 1790: 1049-1058.

Mencke R, Hillebrands JL, consortium N. The role of the anti-ageing protein Klotho in vascular physiology and pathophysiology. Ageing Res Rev 2017; 35: 124-146.

Hu MC, Shi M, Zhang J, Quinones H, Griffith C, Kuro-o $\mathbf{M}$, et al. Klotho deficiency causes vascular calcification in chronic kidney disease. J Am Soc Nephrol 201 1; 22: 124-136.

Mencke R, Umbach AT, Wiggenhauser LM, VoelkI J, Olauson H, Harms G, et al. Klotho deficiency induces arteriolar hyalinosis in a trade-off with vascular calcification. Am J Pathol 2019; 189: 2503-2515.

Zhou X, Chen K, Lei H, Sun Z. Klotho gene deficiency causes salt-sensitive hypertension via monocyte chemotactic 2-mediated inflammation. J Am Soc Nephrol 2015; 26: 121-132.

Lim K, LU TS, Molostvov G, Lee C, Lam FT, Zehnder $\mathbf{D}$, et al. Vascular Klotho deficiency potentiates the development of human artery calcification and mediates resistance to fibroblast growth factor 23 . Circulation 2012; 125: 2243-2255.

\section{Chen YX, Huang C, Duan ZB, Xu CY, Chen Y.} Klotho/FGF23 axis mediates high phosphate-induced vascular calcification in vascular smooth muscle cells via Wnt7b/beta-catenin pathway. Kaohsiung J Med Sci 2019; 35: 393-400.

Lim K, Halim A, Lu T-s, Ashworth A, Chong I. Klotho: A Major Shareholder in Vascular Aging Enterprises. Int J Mol Sci 2019; 20: 4637.

Brown IAM, Diederich L, Good ME, DeLalio LJ, Murphy SA, Cortese-Krott MM, et al. Vascular Smooth Muscle Remodeling in Conductive and Resistance Arteries in Hypertension. Arterioscler Thromb Vasc Biol 2018; 38: 1969-1985.

Johnson JL. Emerging regulators of vascular smooth muscle cell function in the development and progression of atherosclerosis. Cardiovasc Res 2014; 103: 452-460.

Wang $\mathbf{Y}$, Kuro-o $\mathbf{M}$, Sun Z. Klotho gene delivery suppresses Nox2 expression and attenuates oxidative stress in rat aortic 
smooth muscle cells via the CAMP-PKA pathway. Aging Cell 2012; 11: 410-417.

Yu S, Chen Y, Chen S, Ye N, Li Y, Sun Y. Klotho Inhibits Proliferation and Migration of Angiotensin II-Induced Vascular Smooth Muscle Cells (VSMCs) by Modulating NF-kappaB p65, Akt, and Extracellular Signal Regulated Kinase (ERK) Signaling Activities. Med Sci Monit 2018; 24: 4851-4860.

Chen T, Mao H, Chen C, Wu L, Wang N, Zhao $X$, et al. The Role and Mechanism of alpha-Klotho in the Calcification of Rat Aortic Vascular Smooth Muscle Cells. Biomed Res Int 2015; 2015: 194362.

Zhou L, Li Y, Zhou D, Tan RJ, Liu Y. Loss of Klotho contributes to kidney injury by derepression of Wnt/beta-catenin signaling. J Am Soc Nephrol 2013; 24: 771-785.

Ruiz-Ortega $M$, Lorenzo $O$, Suzuki $Y$, Ruperez $M$, Egido J. Proinflammatory actions of angiotensins. Curr Opin Nephrol Hypertens 2001; 10: 321-329.

\section{Tunon J, Ruiz-Ortega $M$ and Egido J.} Regulation of matrix proteins and impact on vascular structure. Curr Hypertens Rep 2000; 2: 106-113.

Rakugi H, Matsukawa N, Ishikawa K, Yang J, Imai $\mathbf{M}$, Ikushima $\mathbf{M}$, et al. Anti-oxidative effect of Klotho on endothelial cells through CAMP activation. Endocrine 2007; 31: 82-87.
Maekawa Y, Ishikawa K, Yasuda O, Oguro R, Hanasaki H, Kida I, et al. Klotho suppresses TNF-alpha-induced expression of adhesion molecules in the endothelium and attenuates NF-kappaB activation. Endocrine 2009; 35: 341-346.

Moe S. Klotho: a master regulator of cardiovascular disease? Circulation 2012; 125: 2181-2183.

Fu Y, Chang Y, Chen S, Li Y, Chen Y, Sun G, et al. BAG3 promotes the phenotypic transformation of primary rat vascular smooth muscle cells via TRAIL. Int J Mol Med 2018; 41: 2917-2926.

Zhao $Y$, Wang $C$, Wang $C$, Hong $X$, Miao J, Liao $Y$, ef al. An essential role for Wnt/beta-catenin signaling in mediating hypertensive heart disease. Sci Rep 2018; 8: 8996.

Xiao L, Xu B, Zhou L, Tan RJ, Zhou D, Fu H, et al. Wnt/beta-catenin regulates blood pressure and kidney injury in rats. Biochim Biophys Acta Mol Basis Dis 2019; 1865: 1313-1322.

Zhao $Y$, Wang $C$, Hong $X$, Miao J, Liao $Y$, Hou FF, et al. Wnt/beta-catenin signaling mediates both heart and kidney injury in type 2 cardiorenal syndrome. Kidney Int 2019; 95: 815-829.

Zhou L, Li Y, Hao S, Zhou D, Tan RJ, Nie J, et al. Multiple genes of the renin-angiotensin system are novel targets of Wnt/beta-catenin signaling. J Am Soc 
Nephrol 2015; 26: 107-120.

MacDonald BT, He X. Frizzled and LRP5/6 receptors for Wnt/ $\beta$-catenin signaling. Cold Spring Harb Perspect Biol 2012; 4: a007880.

Zhou L, Li Y, Zhou D, Tan RJ, Liu Y. Loss of Klotho contributes to kidney injury by derepression of $\mathrm{Wnt} / \mathrm{B}$-catenin signaling. J Am Soc Nephrol 2013; 24: 771-785.

Liu H, Fergusson MM, Castilho RM, Liu J, Cao L, Chen J, et al. Augmented Wnt signaling in a mammalian model of accelerated aging. Science 2007; 317: 803-806.

Zimmerman ZF, Moon RT, Chien AJ. Targeting Wnt pathways in disease. Cold Spring Harb Perspect Biol 2012; 4: a008086.

Chen L, Zhuang J, Singh S, Wang $K$, Xiong $M$, Xu D, et al. XAV939 Inhibits Intima Formation by Decreasing Vascular Smooth Muscle Cell Proliferation and Migration Through Blocking Wnt Signaling. J Cardiovasc Pharmacol 2016; 68: 41 4-424.

Yu L, Meng W, Ding J, Cheng M. Klotho inhibits angiotensin Il-induced cardiomyocyte hypertrophy through suppression of the ATIR/beta catenin pathway. Biochem Biophys Res Commun 2016; 473: 455-461. 